\section{Crystal structure of [ $\left.\mathrm{NaZn}(\mathrm{BTC})\left(\mathrm{H}_{2} \mathrm{O}\right)_{4}\right]$-- $1.5 \mathrm{H}_{2} \mathrm{O}(\mathrm{BTC}=$ benzene-1,3,5-tricarb- oxylate): a heterometallic coordination compound}

\author{
Min Ni, Quanle Li, Hao Chen and Shengqing Li* \\ College of Science, Huazhong Agricultural University, Wuhan, Hubei 430070, \\ People's Republic of China. *Correspondence e-mail: sqingli@mail.hzau.edu.cn
}

Received 28 March 2015; accepted 23 June 2015

Edited by P. Bombicz, Hungarian Academy of Sciences, Hungary

The title coordination polymer, poly[[ $\mu$-aqua-triaqua( $\mu_{3}$-benzene-1,3,5-tricarboxylato)sodiumzinc] sesquihydrate], $\left\{\left[\mathrm{NaZn}\left(\mathrm{C}_{9} \mathrm{H}_{3} \mathrm{O}_{6}\right)\left(\mathrm{H}_{2} \mathrm{O}\right)_{4}\right] \cdot 1.5 \mathrm{H}_{2} \mathrm{O}\right\}_{n}$, was obtained in ionic liquid microemulsion at room temperture by the reaction of benzene-1,3,5-tricarboxylic acid ( $\left.\mathrm{H}_{3} \mathrm{BTC}\right)$ with $\mathrm{Zn}\left(\mathrm{NO}_{3}\right)_{2} \cdot 6 \mathrm{H}_{2} \mathrm{O}$ in the presence of $\mathrm{NaOH}$. The asymmetric unit comprises two $\mathrm{Na}^{+}$ions (each located on an inversion centre), one $\mathrm{Zn}^{2+}$ ion, one BTC ligand, four coordinating water molecules and two solvent water molecules, one of which is disordered about an inversion centre and shows halfoccupation. The $\mathrm{Zn}^{2+}$ cation is five-coordinated by two carboxylate $\mathrm{O}$ atoms from two different BTC ligands and three coordinating $\mathrm{H}_{2} \mathrm{O}$ molecules; the $\mathrm{Zn}-\mathrm{O}$ bond lengths are in the range 1.975 (2)-2.058 (3) $\AA$. The $\mathrm{Na}^{+}$cations are sixcoordinated but have different arrangements of the ligands: one is bound to two carboxylate $\mathrm{O}$ atoms of two BTC ligands and four $\mathrm{O}$ atoms from four coordinating $\mathrm{H}_{2} \mathrm{O}$ molecules while the other is bound by four carboxylate $\mathrm{O}$ atoms from four BTC linkers and two $\mathrm{O}$ atoms of coordinating $\mathrm{H}_{2} \mathrm{O}$ molecules. The completely deprotonated BTC ligand acts as a bridging ligand binding the $\mathrm{Zn}^{2+}$ atom and $\mathrm{Na}^{+}$ions, forming a layered structure extending parallel to (100). An intricate network of $\mathrm{O}-\mathrm{H} \cdots \mathrm{O}$ hydrogen bonds is present within and between the layers.

Keywords: crystal structure; heterometallic coordination compound; benzene-1,3,5-tricarboxylic acid; hydrogen bonding.

CCDC reference: 1055450

\section{Related literature}

For general background to heterometallic coordination compounds, see: Stock \& Biswas (2012); Gao et al. (2005); Zhou et al. (2012). For details of the synthesis, see: Shang et al. (2013); Fu et al. (2011). For the potential application of this compound, see: Huang et al. (2014).

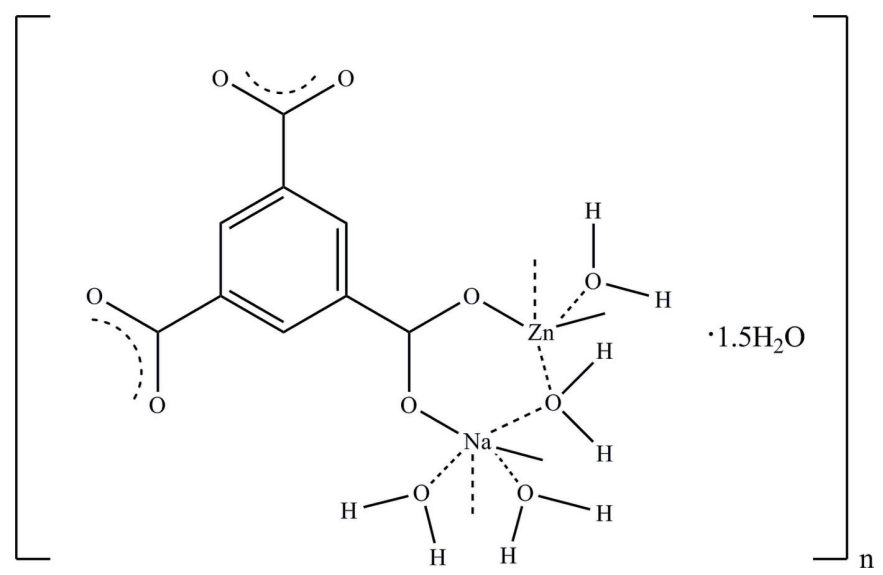

\section{Experimental}

\subsection{Crystal data}

$\left[\mathrm{NaZn}\left(\mathrm{C}_{9} \mathrm{H}_{3} \mathrm{O}_{6}\right)\left(\mathrm{H}_{2} \mathrm{O}\right)_{4}\right] \cdot 1.5 \mathrm{H}_{2} \mathrm{O}$

$M_{r}=394.56$

Triclinic, $P \overline{1}$

$a=7.0980$ (11) $\AA$

$b=9.8000(16) \AA$

$c=11.2043(17) \AA$

$\alpha=66.923$ (2)

$\beta=73.598$ (2)

$$
\begin{aligned}
& \gamma=84.720(3)^{\circ} \\
& V=687.68(19) \AA^{3} \\
& Z=2 \\
& \text { Mo } K \alpha \text { radiation } \\
& \mu=1.88 \mathrm{~mm}^{-1} \\
& T=296 \mathrm{~K} \\
& 0.05 \times 0.03 \times 0.02 \mathrm{~mm}
\end{aligned}
$$

\subsection{Data collection}

Bruker APEXII CCD diffractometer

Absorption correction: multi-scan (SADABS; Bruker, 2009)

$T_{\min }=0.912, T_{\max }=0.963$

7585 measured reflections 4331 independent reflections 2567 reflections with $I>2 \sigma(I)$ $R_{\text {int }}=0.051$

\subsection{Refinement}

$R\left[F^{2}>2 \sigma\left(F^{2}\right)\right]=0.051$

$w R\left(F^{2}\right)=0.113$

$S=0.97$

4331 reflections

214 parameters

$\mathrm{H}$-atom parameters constrained

$\Delta \rho_{\max }=0.79 \mathrm{e} \AA^{-3}$

$\Delta \rho_{\min }=-0.69 \mathrm{e} \AA^{-3}$

Table 1

Hydrogen-bond geometry $\left(\AA \mathrm{A}^{\circ}\right)$.

\begin{tabular}{lllll}
\hline$D-\mathrm{H} \cdots A$ & $D-\mathrm{H}$ & $\mathrm{H} \cdots A$ & $D \cdots A$ & $D-\mathrm{H} \cdots A$ \\
\hline $\mathrm{O} 7-\mathrm{H} 7 A \cdots \mathrm{O}^{\mathrm{i}}$ & 0.82 & 1.79 & $2.587(4)$ & 162 \\
$\mathrm{O} 7-\mathrm{H} 7 B \cdots \mathrm{O} 12^{\mathrm{ii}}$ & 0.82 & 1.93 & $2.740(4)$ & 172 \\
$\mathrm{O} 8-\mathrm{H} 8 A \cdots \mathrm{O} 10$ & 0.82 & 2.40 & $3.114(5)$ & 146 \\
$\mathrm{O} 8-\mathrm{H} 8 A \cdots \mathrm{O} 11$ & 0.82 & 1.98 & $2.672(8)$ & 142 \\
$\mathrm{O} 8-\mathrm{H} 8 B \cdots 6^{\text {ii }}$ & 0.82 & 2.05 & $2.641(5)$ & 128 \\
$\mathrm{O} 9-\mathrm{H} 9 A \cdots \mathrm{O} 12^{\text {iii }}$ & 0.82 & 1.95 & $2.734(4)$ & 159 \\
$\mathrm{O} 9-\mathrm{H} 9 B \cdots 2^{\text {iv }}$ & 0.82 & 2.01 & $2.823(4)$ & 170 \\
$\mathrm{O}^{2}-\mathrm{H} 10 A \cdots$ O5 $^{\mathrm{v}}$ & 0.82 & 2.06 & $2.719(6)$ & 137
\end{tabular}




\begin{tabular}{|c|c|c|c|c|}
\hline$D-\mathrm{H} \cdots A$ & $D-\mathrm{H}$ & $\mathrm{H} \cdots A$ & $D \cdots A$ & $D-\mathrm{H} \cdots A$ \\
\hline$\overline{\mathrm{O} 10-\mathrm{H} 10 B \cdots \mathrm{O} 9^{\mathrm{v} 1}}$ & 0.82 & 2.31 & $3.079(5)$ & 155 \\
\hline $\mathrm{O} 11-\mathrm{H} 11 A \cdots \mathrm{O} 3^{\mathrm{vii}}$ & 0.85 & 2.03 & $2.835(8)$ & 157 \\
\hline $\mathrm{O} 11-\mathrm{H} 11 B \cdots \mathrm{O}^{\mathrm{v}}$ & 0.85 & 2.27 & $2.866(7)$ & 127 \\
\hline $\mathrm{O} 11-\mathrm{H} 11 B \cdots \mathrm{O} 11^{\mathrm{vii}}$ & 0.85 & 1.33 & $1.973(9)$ & 128 \\
\hline $\mathrm{O} 12-\mathrm{H} 12 A \cdots \mathrm{O} 6$ & 0.82 & 1.86 & $2.652(4)$ & 161 \\
\hline $\mathrm{O} 12-\mathrm{H} 12 B \cdots \mathrm{O} 4^{\mathrm{ix}}$ & 0.82 & 1.97 & $2.787(3)$ & 172 \\
\hline
\end{tabular}

Symmetry codes: (i) $\quad x, y+1, z-1$; $\quad$ (ii) $\quad-x+1,-y+2,-z+1$; $-x+2,-y+2,-z+1$; (iv) $-x+2,-y+2,-z$; (v) $-x+1,-y+1,-z+1$; (vi) $x-1, y, z$; (vii) $x-1, y+1, z$; (viii) $-x,-y+2,-z+1$; (ix) $x, y, z+1$.

Data collection: APEX2 (Bruker, 2009); cell refinement: SAINT-Plus (Bruker, 2009); data reduction: SAINT-Plus; program(s) used to solve structure: SHELXS7 (Sheldrick, 2008); program(s) used to refine structure: SHELXL2013 (Sheldrick, 2015); molecular graphics: OLEX2 (Dolomanov et al., 2009); software used to prepare material for publication: $O L E X 2$.

\section{Acknowledgements}

Financial support by the Fundamental Research Funds for the Central Universities (grant Nos. 2011PY128 and 2014PY053) and the National Undergraduate Training Programs for Innovation and Entrepreneurship (grant No. 2015028) of
Huazhong Agricultural University are gratefully acknowledged. We thank Dr Y. Qu of HZAU and Dr X. G. Meng of $\mathrm{CCNU}$ for their kind assistance with this work.

Supporting information for this paper is available from the IUCr electronic archives (Reference: ZP2017).

\section{References}

Bruker (2009). APEX2, SAINT-Plus and SADABS. Bruker AXS Inc., Madison. Wisconsin, USA.

Dolomanov, O. V., Bourhis, L. J., Gildea, R. J., Howard, J. A. K. \& Puschmann, H. (2009). J. Appl. Cryst. 42, 339-341.

Fu, Y., Su, J., Yang, S. H., Zou, Z. B., Li, G. B., Liao, F. H., Xiong, M. \& Lin, J. H. (2011). Cryst. Growth Des. 11, 2243-2249.

Gao, Y. N., Han, S., Han, B., Li, G., Shen, D., Li, Z., Du, J., Hou, W. \& Zhang, G. (2005). Langmuir, 21, 5681-5684.

Huang, X. Q., Chen, Y. F., Lin, Z. G., Ren, X. Q., Song, Y. N., Xu, Z. Z., Dong, X. M., Li, X. G., Hu, C. W. \& Wang, B. (2014). Chem. Commun. 50, 26242627.

Shang, W. T., Kang, X. C., Ning, H., Zhang, J. L., Zhang, X. G., Wu, Z. H., Mo, G., Xing, X. Q. \& Han, B. (2013). Langmuir, 29, 13168-13174.

Sheldrick, G. M. (2008). Acta Cryst. A64, 112-122.

Sheldrick, G. M. (2015). Acta Cryst. C71, 3-8.

Stock, N. \& Biswas, S. (2012). Chem. Rev. 112, 933-969.

Zhou, H. C., Long, J. R. \& Yaghi, O. M. (2012). Chem. Rev. 112, 673-674. 


\section{supporting information}

Acta Cryst. (2015). E71, m143-m144 [doi:10.1107/S2056989015012001]

\section{Crystal structure of $\left[\mathrm{NaZn}(\mathrm{BTC})\left(\mathrm{H}_{2} \mathrm{O}\right)_{4}\right] \cdot 1.5 \mathrm{H}_{2} \mathrm{O}(\mathrm{BTC}=$ benzene-1,3,5-tri- carboxylate): a heterometallic coordination compound}

\section{Min Ni, Quanle Li, Hao Chen and Shengqing Li}

\section{S1. Synthesis and crystallization}

In the experiment, the microemulsion of desired composition containing water, [Bmim] $\mathrm{PF}_{6}$, and Triton $\mathrm{X}-100$ was prepared using the method reported previously (Gao et al. 2005). $\mathrm{H}_{3} \mathrm{BTC}(0.210 \mathrm{~g}, 1.0 \mathrm{mmol}), \mathrm{NaOH}(0.040 \mathrm{~g}, 1.0 \mathrm{mmol})$ and $\mathrm{Zn}\left(\mathrm{NO}_{3}\right)_{2} \cdot 6 \mathrm{H}_{2} \mathrm{O}(0.298 \mathrm{~g}, 1.0 \mathrm{mmol})$ were added one by one into the microemulsion $(20 \mathrm{~g})$ which was clear and transparent system including $1.444 \mathrm{~g}\left[\mathrm{Bmim}^{-} \mathrm{PF}_{6}, 10.428 \mathrm{~g}\right.$ Triton X-100 and $8.310 \mathrm{~g}$ water. The whole system was stirred continuously for $24 \mathrm{~h}$ at $25^{\circ} \mathrm{C}$. Then, the product crystals were collected by centrifugation at $4500 \mathrm{r} / \mathrm{min}$ and washed with alcohol three times $(3 \times 20 \mathrm{~mL})$ to remove the surfactant and $[\mathrm{Bmim}] \mathrm{PF}_{6}$. Then, the crystals were dried in a vacuum oven at $60^{\circ} \mathrm{C}$ for $24 \mathrm{~h}$. The resulting colorless crystals of the title compound were obtained.

\section{S2. Refinement}

Crystal data, data collection and structure refinement details are summarized in Table 1.

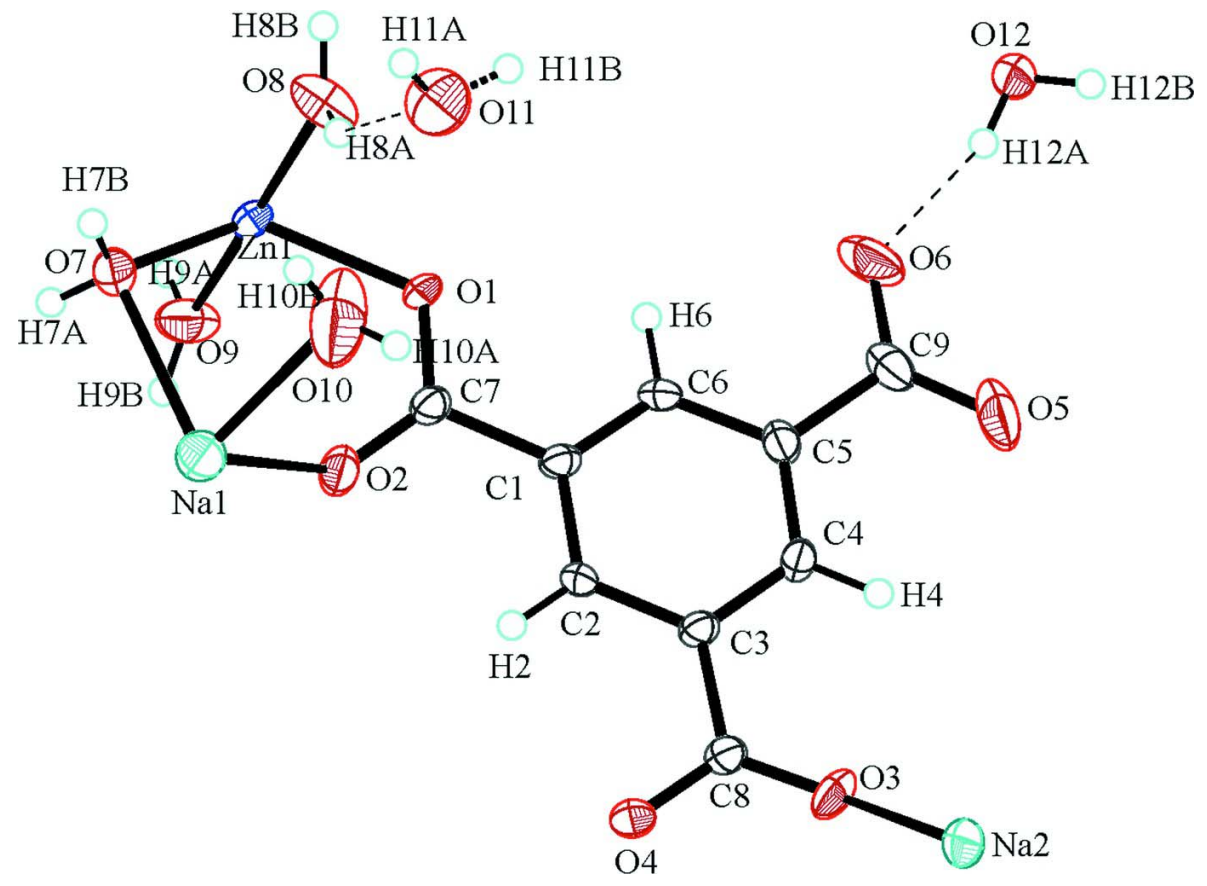

\section{Figure 1}

The molecular structure of the title compound with the atom-numbering scheme and $30 \%$ probability ellipsoids. 


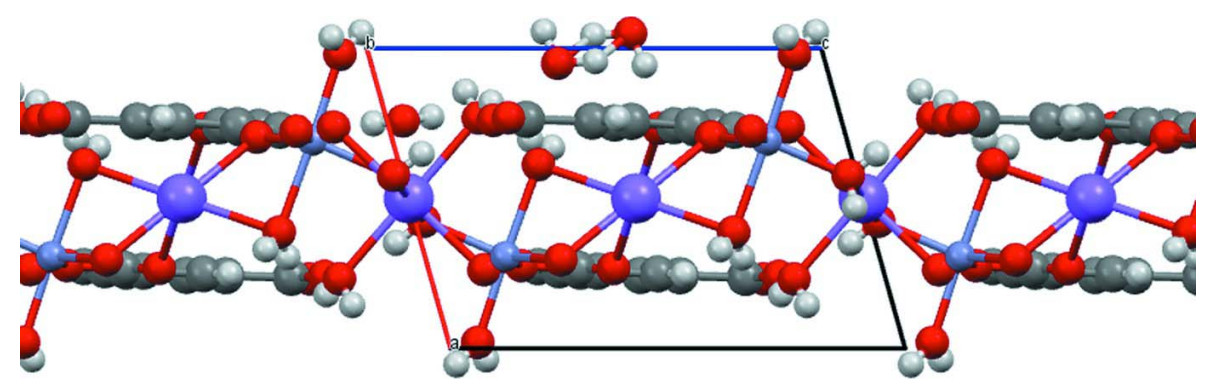

Figure 2

The packing diagram viewed along the $b$ axis.

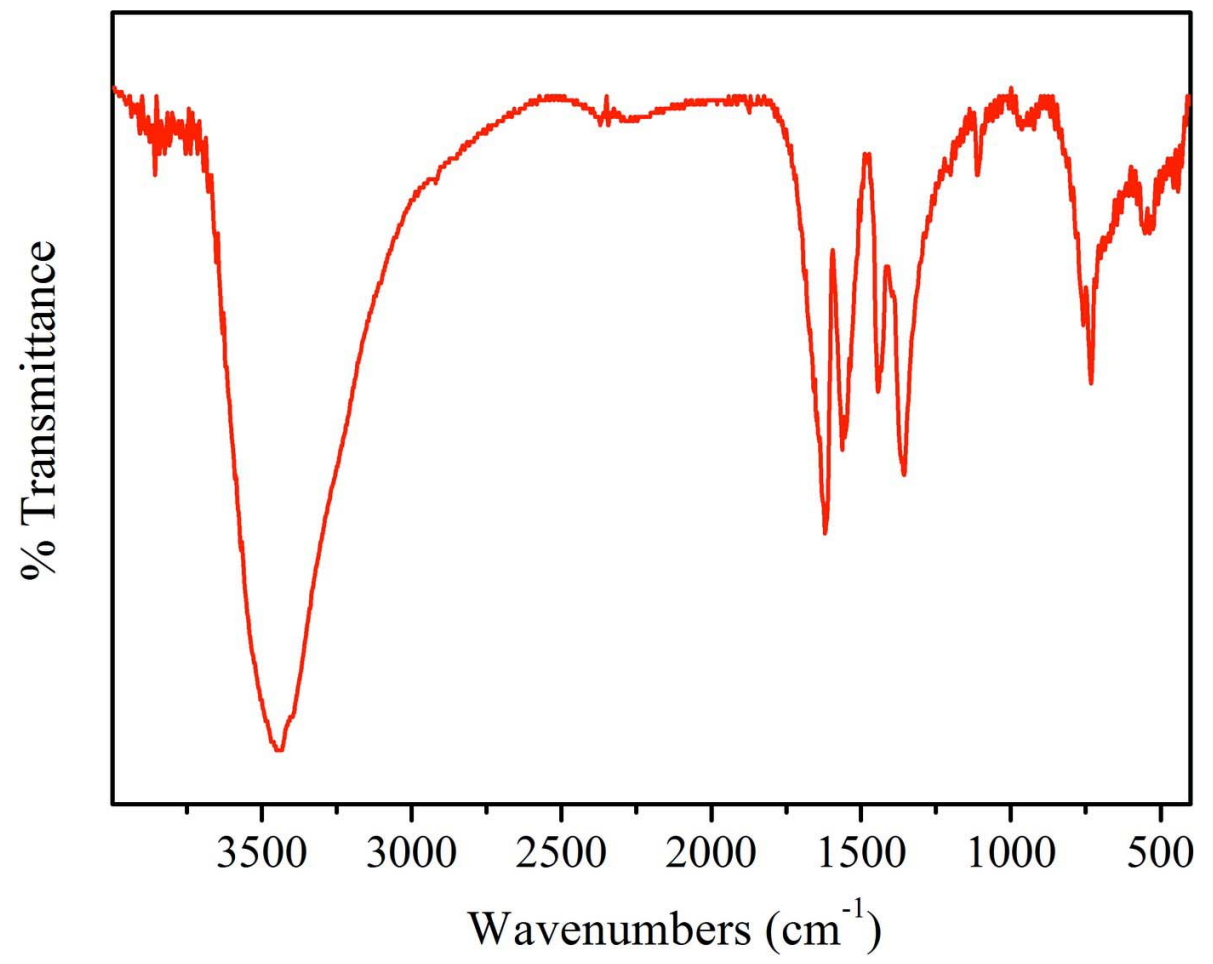

Figure 3

The FT-IR spectrum of the title compound. 


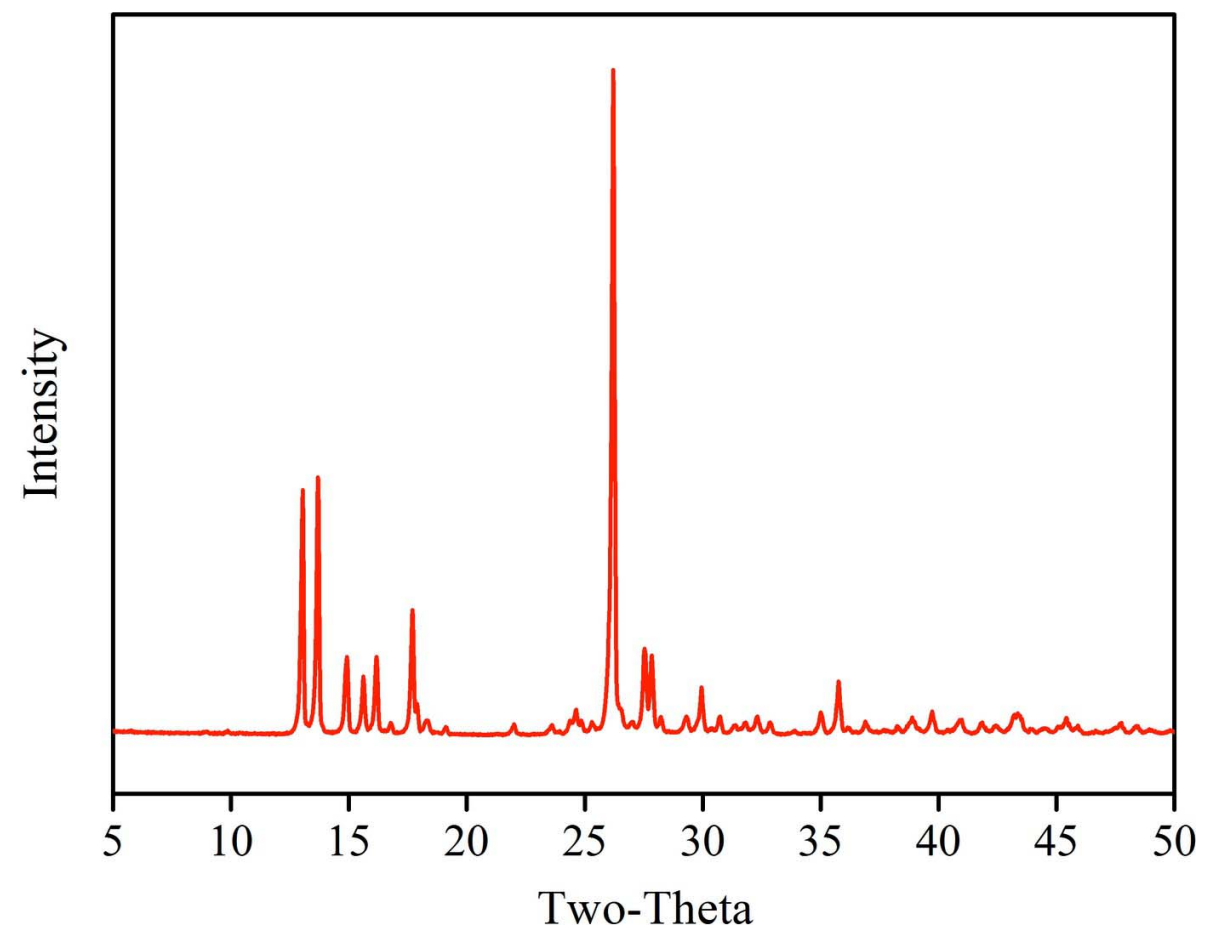

Figure 4

The XRD pattern of the title compound.

Poly[ $\left[\mu\right.$-aqua-triaqua $\left(\mu_{3}\right.$-benzene-1,3,5-tricarboxylato $)$ sodiumzinc $]$ sesquihydrate $]$

\section{Crystal data}

$\left[\mathrm{NaZn}\left(\mathrm{C}_{9} \mathrm{H}_{3} \mathrm{O}_{6}\right)\left(\mathrm{H}_{2} \mathrm{O}\right)_{4}\right] \cdot 1.5 \mathrm{H}_{2} \mathrm{O}$

$M_{r}=394.56$

Triclinic, $P \overline{1}$

$a=7.0980(11) \AA$

$b=9.8000(16) \AA$

$c=11.2043(17) \AA$

$\alpha=66.923(2)^{\circ}$

$\beta=73.598(2)^{\circ}$

$\gamma=84.720(3)^{\circ}$

$V=687.68(19) \AA^{3}$

Data collection

Bruker APEXII CCD

diffractometer

Radiation source: fine-focus sealed tube

$\varphi$ and $\omega$ scans

Absorption correction: multi-scan

(SADABS; Bruker, 2009)

$T_{\text {min }}=0.912, T_{\max }=0.963$

7585 measured reflections
$Z=2$

$F(000)=402$

$D_{\mathrm{x}}=1.906 \mathrm{Mg} \mathrm{m}^{-3}$

Mo $K \alpha$ radiation, $\lambda=0.71073 \AA$

Cell parameters from 1047 reflections

$\theta=2.4-22.5^{\circ}$

$\mu=1.88 \mathrm{~mm}^{-1}$

$T=296 \mathrm{~K}$

Block, colourless

$0.05 \times 0.03 \times 0.02 \mathrm{~mm}$

4331 independent reflections

2567 reflections with $I>2 \sigma(I)$

$R_{\text {int }}=0.051$

$\theta_{\text {max }}=32.0^{\circ}, \theta_{\text {min }}=2.3^{\circ}$

$h=-10 \rightarrow 10$

$k=-14 \rightarrow 14$

$l=-16 \rightarrow 16$ 


\section{Refinement}

Refinement on $F^{2}$

Least-squares matrix: full

$R\left[F^{2}>2 \sigma\left(F^{2}\right)\right]=0.051$

$w R\left(F^{2}\right)=0.113$

$S=0.97$

4331 reflections

214 parameters

0 restraints

Primary atom site location: structure-invariant direct methods
Secondary atom site location: difference Fourier map

Hydrogen site location: mixed

$\mathrm{H}$-atom parameters constrained

$w=1 /\left[\sigma^{2}\left(F_{\mathrm{o}}^{2}\right)+(0.0356 P)^{2}\right]$

where $P=\left(F_{\mathrm{o}}{ }^{2}+2 F_{\mathrm{c}}{ }^{2}\right) / 3$

$(\Delta / \sigma)_{\max }=0.001$

$\Delta \rho_{\max }=0.79 \mathrm{e} \AA^{-3}$

$\Delta \rho_{\min }=-0.69$ e $\AA^{-3}$

Special details

Geometry. All e.s.d.'s (except the e.s.d. in the dihedral angle between two l.s. planes) are estimated using the full covariance matrix. The cell e.s.d.'s are taken into account individually in the estimation of e.s.d.'s in distances, angles and torsion angles; correlations between e.s.d.'s in cell parameters are only used when they are defined by crystal symmetry. An approximate (isotropic) treatment of cell e.s.d.'s is used for estimating e.s.d.'s involving 1.s. planes.

Refinement. olex2_refinement_description 1. Fixed Uiso At 1.2 times of: All C(H) groups At 1.5 times of: All O(H,H) groups 2. Others Fixed Sof: O11(0.5) H11A(0.5) H11B(0.5) 3.a Riding coordinates: O7(H7A,H7B), O8(H8A,H8B), O9(H9A,H9B), O10(H10A,H10B), O12(H12A,H12B) 3.b Free rotating group: O11(H11A,H11B) 3.c Aromatic/amide H refined with riding coordinates: $\mathrm{C} 2(\mathrm{H} 2), \mathrm{C} 4(\mathrm{H} 4), \mathrm{C} 6(\mathrm{H} 6)$

Fractional atomic coordinates and isotropic or equivalent isotropic displacement parameters $\left(\AA^{2}\right)$

\begin{tabular}{|c|c|c|c|c|c|}
\hline & $x$ & $y$ & $z$ & $U_{\text {iso }} * / U_{\text {eq }}$ & Occ. $(<1)$ \\
\hline $\mathrm{Zn} 1$ & $0.69252(6)$ & $1.17043(4)$ & $0.17723(4)$ & $0.02302(13)$ & \\
\hline $\mathrm{Na} 1$ & 0.5000 & 1.0000 & 0.0000 & $0.0322(5)$ & \\
\hline $\mathrm{Na} 2$ & 0.5000 & 0.0000 & 0.5000 & $0.0319(5)$ & \\
\hline $\mathrm{C} 1$ & $0.7424(5)$ & 0.7068 & 0.3327 & $0.0184(7)$ & \\
\hline $\mathrm{C} 2$ & $0.7376(5)$ & 0.5950 & 0.2865 & $0.0186(7)$ & \\
\hline $\mathrm{H} 2$ & 0.7312 & 0.6192 & 0.1988 & $0.022 *$ & \\
\hline $\mathrm{C} 3$ & $0.7423(5)$ & $0.4476(3)$ & $0.3710(3)$ & $0.0188(7)$ & \\
\hline $\mathrm{C} 4$ & $0.7512(5)$ & $0.4130(4)$ & $0.5013(3)$ & $0.0207(7)$ & \\
\hline $\mathrm{H} 4$ & 0.7529 & 0.3140 & 0.5581 & $0.025^{*}$ & \\
\hline $\mathrm{C} 5$ & $0.7576(5)$ & $0.5231(4)$ & $0.5492(3)$ & $0.0211(7)$ & \\
\hline C6 & $0.7566(5)$ & $0.6703(4)$ & $0.4623(3)$ & $0.0204(7)$ & \\
\hline H6 & 0.7657 & 0.7455 & 0.4917 & $0.024 *$ & \\
\hline $\mathrm{C} 7$ & $0.7273(5)$ & $0.8667(4)$ & $0.2423(3)$ & $0.0204(7)$ & \\
\hline $\mathrm{C} 8$ & $0.7345(5)$ & $0.3229(4)$ & $0.3272(3)$ & $0.0206(7)$ & \\
\hline C9 & $0.7632(5)$ & $0.4837(5)$ & $0.6923(4)$ & $0.0291(8)$ & \\
\hline $\mathrm{O} 1$ & $0.7044(4)$ & $0.9607(3)$ & $0.2967(3)$ & $0.0371(7)$ & \\
\hline $\mathrm{O} 2$ & $0.7376(4)$ & 0.8999 & $0.1217(3)$ & $0.0341(6)$ & \\
\hline $\mathrm{O} 3$ & $0.7335(4)$ & 0.1931 (3) & $0.4071(3)$ & $0.0325(6)$ & \\
\hline $\mathrm{O} 4$ & $0.7242(4)$ & 0.3558 & $0.2063(2)$ & $0.0268(6)$ & \\
\hline $\mathrm{O} 5$ & $0.7596(4)$ & $0.3477(4)$ & 0.7661 & $0.0467(8)$ & \\
\hline O6 & $0.7682(4)$ & $0.5863(4)$ & $0.7316(3)$ & $0.0479(8)$ & \\
\hline $\mathrm{O} 7$ & $0.5762(4)$ & $1.2363(3)$ & $0.0195(2)$ & $0.0292(6)$ & \\
\hline H7A & 0.6483 & 1.2811 & -0.0557 & $0.044^{*}$ & \\
\hline H7B & 0.4703 & 1.2778 & 0.0249 & $0.044^{*}$ & \\
\hline $\mathrm{O} 8$ & $0.3875(4)$ & $1.1497(3)$ & 0.3061 & $0.0396(7)$ & \\
\hline
\end{tabular}




\begin{tabular}{|c|c|c|c|c|c|}
\hline $\mathrm{H} 8 \mathrm{~A}$ & 0.3040 & 1.0995 & 0.3031 & $0.059^{*}$ & \\
\hline H8B & 0.3305 & 1.2022 & 0.3464 & $0.059^{*}$ & \\
\hline O9 & $0.9800(4)$ & 1.1943 & $0.0630(3)$ & $0.0382(7)$ & \\
\hline H9A & 1.0364 & 1.2580 & 0.0713 & $0.057^{*}$ & \\
\hline H9B & 1.0536 & 1.1721 & 0.0026 & $0.057^{*}$ & \\
\hline $\mathrm{O} 10$ & $0.2322(5)$ & $0.9423(4)$ & $0.2021(4)$ & $0.0790(13)$ & \\
\hline $\mathrm{H} 10 \mathrm{~A}$ & 0.1808 & 0.8593 & 0.2402 & $0.119^{*}$ & \\
\hline H10B & 0.1445 & 1.0027 & 0.1899 & $0.119^{*}$ & \\
\hline O11 & 0.0497 (9) & $0.9938(8)$ & $0.4107(6)$ & $0.0482(16)$ & 0.5 \\
\hline H11A & -0.0558 & 1.0390 & 0.3993 & $0.072 *$ & 0.5 \\
\hline H11B & 0.0429 & 0.9553 & 0.4942 & $0.072 *$ & 0.5 \\
\hline $\mathrm{O} 12$ & $0.7580(4)$ & 0.6003 & $0.9650(2)$ & $0.0294(6)$ & \\
\hline $\mathrm{H} 12 \mathrm{~A}$ & 0.7717 & 0.5773 & 0.9002 & $0.044 *$ & \\
\hline H12B & 0.7499 & 0.5234 & 1.0318 & $0.044 *$ & \\
\hline
\end{tabular}

Atomic displacement parameters $\left(\AA^{2}\right)$

\begin{tabular}{lllllll}
\hline & $U^{11}$ & $U^{22}$ & $U^{33}$ & $U^{12}$ & $U^{13}$ & $U^{23}$ \\
\hline $\mathrm{Zn} 1$ & $0.0340(2)$ & $0.01306(19)$ & $0.0218(2)$ & $-0.00099(15)$ & $-0.00540(16)$ & $-0.00759(16)$ \\
$\mathrm{Na} 1$ & $0.0424(13)$ & $0.0290(11)$ & $0.0309(12)$ & $0.0027(9)$ & $-0.0169(10)$ & $-0.0128(10)$ \\
$\mathrm{Na} 2$ & $0.0457(13)$ & $0.0204(10)$ & $0.0228(11)$ & $-0.0072(9)$ & $-0.0013(9)$ & $-0.0048(9)$ \\
$\mathrm{C} 1$ & $0.0191(16)$ & $0.0153(15)$ & $0.0210(17)$ & $-0.0019(12)$ & $-0.0009(13)$ & $-0.0096(13)$ \\
$\mathrm{C} 2$ & $0.0267(18)$ & $0.0163(16)$ & $0.0141(16)$ & $-0.0003(13)$ & $-0.0044(13)$ & $-0.0078(13)$ \\
$\mathrm{C} 3$ & $0.0211(17)$ & $0.0145(15)$ & $0.0205(17)$ & $-0.0018(12)$ & $-0.0023(13)$ & $-0.0081(13)$ \\
$\mathrm{C} 4$ & $0.0239(18)$ & $0.0150(16)$ & $0.0184(17)$ & $0.0016(13)$ & $-0.0045(13)$ & $-0.0025(13)$ \\
$\mathrm{C} 5$ & $0.0217(17)$ & $0.0228(17)$ & $0.0150(17)$ & $0.0005(13)$ & $-0.0033(13)$ & $-0.0045(14)$ \\
$\mathrm{C} 6$ & $0.0259(18)$ & $0.0190(16)$ & $0.0194(17)$ & $0.0014(13)$ & $-0.0039(13)$ & $-0.0123(14)$ \\
$\mathrm{C} 7$ & $0.0221(17)$ & $0.0146(16)$ & $0.0221(18)$ & $-0.0014(13)$ & $-0.0017(14)$ & $-0.0070(14)$ \\
$\mathrm{C} 8$ & $0.0229(17)$ & $0.0170(16)$ & $0.0221(18)$ & $0.0007(13)$ & $-0.0047(14)$ & $-0.0089(14)$ \\
$\mathrm{C} 9$ & $0.0241(19)$ & $0.044(2)$ & $0.0187(19)$ & $0.0110(16)$ & $-0.0063(15)$ & $-0.0131(18)$ \\
O1 & $0.071(2)$ & $0.0100(12)$ & $0.0212(14)$ & $0.0004(12)$ & $0.0018(13)$ & $-0.0062(11)$ \\
O2 & $0.0607(19)$ & $0.0187(13)$ & $0.0253(15)$ & $0.0030(12)$ & $-0.0202(13)$ & $-0.0053(11)$ \\
O3 & $0.0481(17)$ & $0.0124(12)$ & $0.0351(16)$ & $0.0001(11)$ & $-0.0128(12)$ & $-0.0058(11)$ \\
O4 & $0.0411(15)$ & $0.0191(12)$ & $0.0235(14)$ & $-0.0019(10)$ & $-0.0079(11)$ & $-0.0117(11)$ \\
O5 & $0.0529(19)$ & $0.053(2)$ & $0.0203(15)$ & $0.0040(15)$ & $-0.0114(13)$ & $0.0006(14)$ \\
O6 & $0.058(2)$ & $0.070(2)$ & $0.0325(17)$ & $0.0229(17)$ & $-0.0228(14)$ & $-0.0346(17)$ \\
O7 & $0.0337(15)$ & $0.0261(14)$ & $0.0213(14)$ & $0.0047(11)$ & $-0.0037(11)$ & $-0.0057(11)$ \\
O8 & $0.0298(15)$ & $0.065(2)$ & $0.0303(16)$ & $-0.0049(13)$ & $-0.0034(12)$ & $-0.0265(15)$ \\
O9 & $0.0386(17)$ & $0.0369(16)$ & $0.0418(18)$ & $-0.0062(12)$ & $0.0029(13)$ & $-0.0260(14)$ \\
O10 & $0.059(2)$ & $0.062(3)$ & $0.068(3)$ & $0.0028(18)$ & $-0.0052(18)$ & $0.016(2)$ \\
O11 & $0.041(4)$ & $0.052(4)$ & $0.052(4)$ & $0.008(3)$ & $-0.018(3)$ & $-0.019(4)$ \\
O12 & $0.0440(16)$ & $0.0224(13)$ & $0.0223(14)$ & $-0.0001(11)$ & $-0.0096(11)$ & $-0.0086(11)$ \\
& & & & & & \\
\hline
\end{tabular}

Geometric parameters $\left(\AA,{ }^{\circ}\right)$

\begin{tabular}{llll}
\hline $\mathrm{Zn} 1-\mathrm{Na} 1$ & $3.6267(5)$ & $\mathrm{C} 3-\mathrm{C} 8$ & $1.495(4)$ \\
$\mathrm{Zn} 1-\mathrm{Na} 2^{\mathrm{i}}$ & $3.2603(6)$ & $\mathrm{C} 4-\mathrm{H} 4$ & 0.9300 \\
$\mathrm{Zn} 1-\mathrm{O} 1$ & $1.975(2)$ & $\mathrm{C} 4-\mathrm{C} 5$ & $1.390(5)$
\end{tabular}




\begin{tabular}{|c|c|c|c|}
\hline $\mathrm{Zn} 1-\mathrm{O} 4^{\mathrm{i}}$ & $2.009(2)$ & $\mathrm{C} 5-\mathrm{C} 6$ & $1.390(4)$ \\
\hline $\mathrm{Zn} 1-\mathrm{O} 7$ & $2.013(2)$ & $\mathrm{C} 5-\mathrm{C} 9$ & $1.506(5)$ \\
\hline $\mathrm{Zn} 1-\mathrm{O} 8$ & $2.214(3)$ & C6- $\mathrm{H} 6$ & 0.9300 \\
\hline $\mathrm{Zn} 1-\mathrm{O} 9$ & $2.058(3)$ & $\mathrm{C} 7-\mathrm{O} 1$ & $1.267(4)$ \\
\hline $\mathrm{Na} 1-\mathrm{Zn} 1^{\mathrm{ii}}$ & $3.6267(5)$ & $\mathrm{C} 7-\mathrm{O} 2$ & $1.242(4)$ \\
\hline $\mathrm{Na} 1-\mathrm{O} 2$ & $2.369(3)$ & $\mathrm{C} 8-\mathrm{O} 3$ & $1.235(4)$ \\
\hline $\mathrm{Na} 1-\mathrm{O} 2^{\mathrm{ii}}$ & $2.369(3)$ & $\mathrm{C} 8-\mathrm{O} 4$ & $1.286(4)$ \\
\hline $\mathrm{Na} 1-\mathrm{O} 7$ & $2.529(3)$ & $\mathrm{C} 9-\mathrm{O} 5$ & $1.262(5)$ \\
\hline $\mathrm{Na} 1-\mathrm{O} 7^{\mathrm{ii}}$ & $2.529(3)$ & $\mathrm{C} 9-\mathrm{O} 6$ & $1.252(5)$ \\
\hline $\mathrm{Na} 1-\mathrm{O} 10$ & $2.413(3)$ & $\mathrm{O} 1-\mathrm{Na} 2^{\mathrm{i}}$ & $2.482(2)$ \\
\hline $\mathrm{Na} 1-\mathrm{O} 10^{\mathrm{ii}}$ & $2.413(3)$ & $\mathrm{O} 4-\mathrm{Zn} 1^{\text {iv }}$ & $2.009(2)$ \\
\hline $\mathrm{Na} 2-\mathrm{Zn} 1^{\mathrm{iii}}$ & $3.2603(6)$ & $\mathrm{O} 7-\mathrm{H} 7 \mathrm{~A}$ & 0.8201 \\
\hline $\mathrm{Na} 2-\mathrm{Zn} 1^{\text {iv }}$ & $3.2603(6)$ & $\mathrm{O} 7-\mathrm{H} 7 \mathrm{~B}$ & 0.8201 \\
\hline $\mathrm{Na} 2-\mathrm{O} 1^{\mathrm{iv}}$ & $2.482(2)$ & $\mathrm{O} 8-\mathrm{Na} 2^{\mathrm{i}}$ & $2.403(3)$ \\
\hline $\mathrm{Na} 2-\mathrm{O} 1^{\mathrm{iii}}$ & $2.482(2)$ & $\mathrm{O} 8-\mathrm{H} 8 \mathrm{~A}$ & 0.8200 \\
\hline $\mathrm{Na} 2-\mathrm{O} 3$ & $2.339(3)$ & $\mathrm{O} 8-\mathrm{H} 8 \mathrm{~B}$ & 0.8201 \\
\hline $\mathrm{Na} 2-\mathrm{O}^{\mathrm{v}}$ & $2.339(3)$ & O9-H9A & 0.8199 \\
\hline $\mathrm{Na} 2-\mathrm{O} 8^{\mathrm{iii}}$ & $2.403(3)$ & O9—H9B & 0.8200 \\
\hline $\mathrm{Na} 2-\mathrm{O} 8^{\mathrm{iv}}$ & $2.403(3)$ & $\mathrm{O} 10-\mathrm{H} 10 \mathrm{~A}$ & 0.8200 \\
\hline $\mathrm{C} 1-\mathrm{C} 2$ & $1.389(4)$ & $\mathrm{O} 10-\mathrm{H} 10 \mathrm{~B}$ & 0.8200 \\
\hline $\mathrm{C} 1-\mathrm{C} 6$ & $1.384(4)$ & O11-H11A & 0.8500 \\
\hline $\mathrm{C} 1-\mathrm{C} 7$ & $1.509(4)$ & O11-H11B & 0.8500 \\
\hline $\mathrm{C} 2-\mathrm{H} 2$ & 0.9300 & $\mathrm{O} 12-\mathrm{H} 12 \mathrm{~A}$ & 0.8203 \\
\hline $\mathrm{C} 2-\mathrm{C} 3$ & $1.386(4)$ & $\mathrm{O} 12-\mathrm{H} 12 \mathrm{~B}$ & 0.8200 \\
\hline $\mathrm{C} 3-\mathrm{C} 4$ & $1.382(4)$ & & \\
\hline $\mathrm{Na} 2-\mathrm{Zn} 1-\mathrm{Na} 1$ & $108.751(15)$ & $\mathrm{O} 3^{\mathrm{v}}-\mathrm{Na} 2-\mathrm{O}^{\mathrm{iii}}$ & $81.75(9)$ \\
\hline $\mathrm{O} 1-\mathrm{Zn} 1-\mathrm{Na} 1$ & $81.47(8)$ & $\mathrm{O} 3-\mathrm{Na} 2-\mathrm{O} 8^{\mathrm{iii}}$ & $98.25(9)$ \\
\hline $\mathrm{O} 1-\mathrm{Zn} 1-\mathrm{Na} 2^{\mathrm{i}}$ & $49.45(7)$ & $\mathrm{O} 3-\mathrm{Na} 2-\mathrm{O}^{\mathrm{iv}}$ & $81.75(9)$ \\
\hline $\mathrm{O} 1-\mathrm{Zn} 1-\mathrm{O} 4^{\mathrm{i}}$ & $129.50(11)$ & 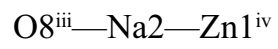 & $137.24(6)$ \\
\hline $\mathrm{O} 1-\mathrm{Zn} 1-\mathrm{O} 7$ & $123.49(11)$ & $\mathrm{O} 8^{\mathrm{iv}}-\mathrm{Na} 2-\mathrm{Zn} 1^{\mathrm{iii}}$ & $137.24(6)$ \\
\hline $\mathrm{O} 1-\mathrm{Zn} 1-\mathrm{O} 8$ & 83.14 (11) & $\mathrm{O} 8^{\mathrm{iii}}-\mathrm{Na} 2-\mathrm{Zn} 1^{\mathrm{iii}}$ & $42.76(6)$ \\
\hline $\mathrm{O} 1-\mathrm{Zn} 1-\mathrm{O} 9$ & $97.22(11)$ & $\mathrm{O} 8^{\mathrm{iv}}-\mathrm{Na} 2-\mathrm{Zn} 1^{\mathrm{iv}}$ & $42.76(6)$ \\
\hline 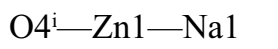 & $147.26(7)$ & 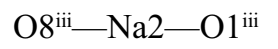 & $69.50(9)$ \\
\hline 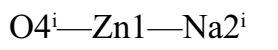 & $89.66(7)$ & 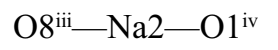 & $110.50(9)$ \\
\hline $\mathrm{O} 4 \mathrm{i}-\mathrm{Zn} 1-\mathrm{O} 7$ & $105.44(10)$ & $\mathrm{O}^{\mathrm{iv}}-\mathrm{Na} 2-\mathrm{O}^{\mathrm{iv}}$ & $69.50(9)$ \\
\hline $\mathrm{O} 4^{\mathrm{i}}-\mathrm{Zn} 1-\mathrm{O} 8$ & $88.15(11)$ & $\mathrm{O} 8^{\mathrm{iv}}-\mathrm{Na} 2-\mathrm{O} 1^{\mathrm{iii}}$ & $110.50(9)$ \\
\hline $\mathrm{O} 4 \mathrm{i}-\mathrm{Zn} 1-\mathrm{O} 9$ & $89.49(10)$ & 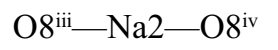 & 180.0 \\
\hline $\mathrm{O} 7-\mathrm{Zn} 1-\mathrm{Na} 1$ & $42.24(7)$ & $\mathrm{C} 2-\mathrm{C} 1-\mathrm{C} 7$ & $119.8(3)$ \\
\hline $\mathrm{O} 7-\mathrm{Zn} 1-\mathrm{Na} 2^{\mathrm{i}}$ & $131.91(7)$ & $\mathrm{C} 6-\mathrm{C} 1-\mathrm{C} 2$ & $119.7(3)$ \\
\hline O7-Zn1-O8 & $86.91(10)$ & $\mathrm{C} 6-\mathrm{C} 1-\mathrm{C} 7$ & $120.6(3)$ \\
\hline $\mathrm{O} 7-\mathrm{Zn} 1-\mathrm{O} 9$ & 95.17 (11) & $\mathrm{C} 1-\mathrm{C} 2-\mathrm{H} 2$ & 119.9 \\
\hline $\mathrm{O} 8-\mathrm{Zn} 1-\mathrm{Na} 1$ & $85.30(7)$ & $\mathrm{C} 3-\mathrm{C} 2-\mathrm{C} 1$ & $120.1(3)$ \\
\hline $\mathrm{O} 8-\mathrm{Zn} 1-\mathrm{Na} 2^{\mathrm{i}}$ & $47.48(7)$ & $\mathrm{C} 3-\mathrm{C} 2-\mathrm{H} 2$ & 119.9 \\
\hline $\mathrm{O} 9-\mathrm{Zn} 1-\mathrm{Na} 1$ & $97.50(8)$ & $\mathrm{C} 2-\mathrm{C} 3-\mathrm{C} 8$ & $122.3(3)$ \\
\hline $\mathrm{O} 9-\mathrm{Zn} 1-\mathrm{Na} 2^{\mathrm{i}}$ & $131.02(8)$ & $\mathrm{C} 4-\mathrm{C} 3-\mathrm{C} 2$ & $119.4(3)$ \\
\hline $\mathrm{O} 9-\mathrm{Zn} 1-\mathrm{O} 8$ & $177.20(11)$ & $\mathrm{C} 4-\mathrm{C} 3-\mathrm{C} 8$ & $118.2(3)$ \\
\hline $\mathrm{Zn} 1-\mathrm{Na} 1-\mathrm{Zn} 1^{\mathrm{ii}}$ & 180.0 & $\mathrm{C} 3-\mathrm{C} 4-\mathrm{H} 4$ & 119.3 \\
\hline
\end{tabular}




\begin{tabular}{|c|c|c|c|}
\hline $\mathrm{O} 2-\mathrm{Na} 1-\mathrm{Zn} 1$ & $53.49(6)$ & $\mathrm{C} 3-\mathrm{C} 4-\mathrm{C} 5$ & $121.4(3)$ \\
\hline $\mathrm{O} 2^{\mathrm{ii}-}-\mathrm{Na} 1-\mathrm{Zn} 1^{\mathrm{ii}}$ & $53.49(6)$ & $\mathrm{C} 5-\mathrm{C} 4-\mathrm{H} 4$ & 119.3 \\
\hline 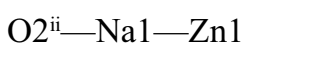 & $126.51(6)$ & $\mathrm{C} 4-\mathrm{C} 5-\mathrm{C} 9$ & $120.8(3)$ \\
\hline $\mathrm{O} 2-\mathrm{Na} 1-\mathrm{Zn} 1^{\mathrm{ii}}$ & $126.51(6)$ & $\mathrm{C} 6-\mathrm{C} 5-\mathrm{C} 4$ & $118.3(3)$ \\
\hline $\mathrm{O} 2{ }^{\mathrm{ii}}-\mathrm{Na} 1-\mathrm{O} 2$ & 180.0 & $\mathrm{C} 6-\mathrm{C} 5-\mathrm{C} 9$ & $120.8(3)$ \\
\hline $\mathrm{O} 2^{\mathrm{ii}}-\mathrm{Na} 1-\mathrm{O} 7^{\mathrm{ii}}$ & $83.14(8)$ & $\mathrm{C} 1-\mathrm{C} 6-\mathrm{C} 5$ & $121.0(3)$ \\
\hline $\mathrm{O} 2-\mathrm{Na} 1-\mathrm{O} 7$ & $83.14(8)$ & $\mathrm{C} 1-\mathrm{C} 6-\mathrm{H} 6$ & 119.5 \\
\hline 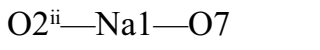 & $96.86(8)$ & $\mathrm{C} 5-\mathrm{C} 6-\mathrm{H} 6$ & 119.5 \\
\hline $\mathrm{O} 2-\mathrm{Na} 1-\mathrm{O}^{\mathrm{ii}}$ & $96.86(8)$ & $\mathrm{O} 1-\mathrm{C} 7-\mathrm{C} 1$ & $116.3(3)$ \\
\hline $\mathrm{O} 2-\mathrm{Na} 1-\mathrm{O} 10^{\mathrm{ii}}$ & $86.91(11)$ & $\mathrm{O} 2-\mathrm{C} 7-\mathrm{C} 1$ & $120.1(3)$ \\
\hline $\mathrm{O} 2^{\mathrm{ii}}-\mathrm{Na} 1-\mathrm{O} 10^{\mathrm{ii}}$ & 93.09 (11) & $\mathrm{O} 2-\mathrm{C} 7-\mathrm{O} 1$ & $123.6(3)$ \\
\hline $\mathrm{O} 2^{\mathrm{ii}}-\mathrm{Na} 1-\mathrm{O} 10$ & $86.91(11)$ & $\mathrm{O} 3-\mathrm{C} 8-\mathrm{C} 3$ & $120.1(3)$ \\
\hline $\mathrm{O} 2-\mathrm{Na} 1-\mathrm{O} 10$ & $93.09(11)$ & $\mathrm{O} 3-\mathrm{C} 8-\mathrm{O} 4$ & $122.0(3)$ \\
\hline 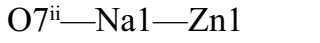 & $147.65(5)$ & $\mathrm{O} 4-\mathrm{C} 8-\mathrm{C} 3$ & $117.9(3)$ \\
\hline $\mathrm{O} 7-\mathrm{Na} 1-\mathrm{Zn} 1$ & $32.36(5)$ & $\mathrm{O} 5-\mathrm{C} 9-\mathrm{C} 5$ & $117.2(4)$ \\
\hline $\mathrm{O} 7^{\mathrm{ii}}-\mathrm{Na} 1-\mathrm{Zn} 1^{\mathrm{ii}}$ & $32.35(5)$ & $\mathrm{O} 6-\mathrm{C} 9-\mathrm{C} 5$ & $118.8(4)$ \\
\hline $\mathrm{O} 7-\mathrm{Na} 1-\mathrm{Zn} 1^{\mathrm{ii}}$ & $147.64(5)$ & $\mathrm{O} 6-\mathrm{C} 9-\mathrm{O} 5$ & $124.0(4)$ \\
\hline $\mathrm{O} 7 \mathrm{ii}-\mathrm{Na} 1-\mathrm{O} 7$ & 180.0 & $\mathrm{Zn} 1-\mathrm{O} 1-\mathrm{Na}^{2}$ & $93.35(9)$ \\
\hline $\mathrm{O} 10^{\mathrm{ii}}-\mathrm{Na} 1-\mathrm{Zn} 1$ & $99.48(11)$ & $\mathrm{C} 7-\mathrm{O} 1-\mathrm{Zn} 1$ & $116.3(2)$ \\
\hline $\mathrm{O} 10-\mathrm{Na} 1-\mathrm{Zn} 1^{\mathrm{ii}}$ & $99.48(11)$ & $\mathrm{C} 7-\mathrm{O} 1-\mathrm{Na} 2^{\mathrm{i}}$ & $140.1(2)$ \\
\hline $\mathrm{O} 10-\mathrm{Na} 1-\mathrm{Zn} 1$ & $80.52(11)$ & $\mathrm{C} 7-\mathrm{O} 2-\mathrm{Na} 1$ & $130.6(2)$ \\
\hline $\mathrm{O} 10^{\mathrm{ii}}-\mathrm{Na} 1-\mathrm{Zn} 1^{\mathrm{ii}}$ & $80.52(11)$ & $\mathrm{C} 8-\mathrm{O} 3-\mathrm{Na} 2$ & $132.2(2)$ \\
\hline $\mathrm{O} 10-\mathrm{Na} 1-\mathrm{O} 7^{\mathrm{ii}}$ & $89.62(12)$ & $\mathrm{C} 8-\mathrm{O} 4-\mathrm{Zn} 1^{\mathrm{iv}}$ & $110.2(2)$ \\
\hline $\mathrm{O} 10-\mathrm{Na} 1-\mathrm{O} 7$ & $90.38(12)$ & $\mathrm{Zn} 1-\mathrm{O} 7-\mathrm{Na} 1$ & $105.40(10)$ \\
\hline $\mathrm{O} 10^{\mathrm{ii}}-\mathrm{Na} 1-\mathrm{O} 7^{\mathrm{ii}}$ & $90.38(12)$ & $\mathrm{Zn} 1-\mathrm{O} 7-\mathrm{H} 7 \mathrm{~A}$ & 117.9 \\
\hline $\mathrm{O} 10^{\mathrm{ii}}-\mathrm{Na} 1-\mathrm{O} 7$ & $89.62(12)$ & $\mathrm{Zn} 1-\mathrm{O} 7-\mathrm{H} 7 \mathrm{~B}$ & 118.8 \\
\hline $\mathrm{O} 10^{\mathrm{ii}}-\mathrm{Na} 1-\mathrm{O} 10$ & 180.0 & $\mathrm{Na} 1-\mathrm{O} 7-\mathrm{H} 7 \mathrm{~A}$ & 101.6 \\
\hline $\mathrm{Zn} 1^{\mathrm{iii}}-\mathrm{Na} 2-\mathrm{Zn} 1^{\mathrm{iv}}$ & 180.0 & $\mathrm{Na} 1-\mathrm{O} 7-\mathrm{H} 7 \mathrm{~B}$ & 102.9 \\
\hline $\mathrm{O} 1^{\mathrm{iii}}-\mathrm{Na} 2-\mathrm{Zn} 1^{\mathrm{iv}}$ & $142.80(5)$ & $\mathrm{H} 7 \mathrm{~A}-\mathrm{O} 7-\mathrm{H} 7 \mathrm{~B}$ & 107.7 \\
\hline $\mathrm{O} 1^{\mathrm{iv}}-\mathrm{Na} 2-\mathrm{Zn} 1^{\text {iv }}$ & $37.20(5)$ & $\mathrm{Zn} 1-\mathrm{O} 8-\mathrm{Na} 2^{\mathrm{i}}$ & $89.76(9)$ \\
\hline $\mathrm{O} 1^{\mathrm{iv}}-\mathrm{Na} 2-\mathrm{Zn} 1^{\mathrm{iii}}$ & $142.80(5)$ & $\mathrm{Zn} 1-\mathrm{O} 8-\mathrm{H} 8 \mathrm{~A}$ & 121.4 \\
\hline 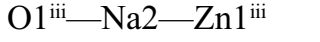 & $37.20(5)$ & $\mathrm{Zn} 1-\mathrm{O} 8-\mathrm{H} 8 \mathrm{~B}$ & 129.0 \\
\hline $\mathrm{O} 1^{\mathrm{iv}}-\mathrm{Na} 2-\mathrm{O} 1^{\mathrm{iii}}$ & 180.0 & $\mathrm{Na} 2^{\mathrm{i}}-\mathrm{O} 8-\mathrm{H} 8 \mathrm{~A}$ & 106.3 \\
\hline $\mathrm{O}^{\mathrm{v}}-\mathrm{Na} 2-\mathrm{Zn} 1^{\mathrm{iv}}$ & $123.65(7)$ & $\mathrm{Na} 2-\mathrm{O} 8-\mathrm{H} 8 \mathrm{~B}$ & 89.2 \\
\hline $\mathrm{O} 3-\mathrm{Na} 2-\mathrm{Zn} 1^{\mathrm{iv}}$ & $56.35(7)$ & $\mathrm{H} 8 \mathrm{~A}-\mathrm{O} 8-\mathrm{H} 8 \mathrm{~B}$ & 107.7 \\
\hline $\mathrm{O} 3{ }^{\mathrm{v}}-\mathrm{Na} 2-\mathrm{Zn} 1^{\mathrm{iii}}$ & $56.35(7)$ & $\mathrm{Zn} 1-\mathrm{O} 9-\mathrm{H} 9 \mathrm{~A}$ & 109.9 \\
\hline $\mathrm{O} 3-\mathrm{Na} 2-\mathrm{Zn} 1^{i \mathrm{ii}}$ & $123.65(7)$ & $\mathrm{Zn} 1-\mathrm{O} 9-\mathrm{H} 9 \mathrm{~B}$ & 141.4 \\
\hline $\mathrm{O} 3-\mathrm{Na} 2-\mathrm{O} 1^{\mathrm{iii}}$ & $102.11(9)$ & $\mathrm{H} 9 \mathrm{~A}-\mathrm{O} 9-\mathrm{H} 9 \mathrm{~B}$ & 107.7 \\
\hline $\mathrm{O} 3-\mathrm{Na} 2-\mathrm{O} 1^{\mathrm{iv}}$ & $77.89(9)$ & $\mathrm{Na} 1-\mathrm{O} 10-\mathrm{H} 10 \mathrm{~A}$ & 120.4 \\
\hline $\mathrm{O}^{\mathrm{v}}-\mathrm{Na} 2-\mathrm{O} 1^{\mathrm{iii}}$ & $77.89(9)$ & $\mathrm{Na} 1-\mathrm{O} 10-\mathrm{H} 10 \mathrm{~B}$ & 110.7 \\
\hline $\mathrm{O}^{\mathrm{v}}-\mathrm{Na} 2-\mathrm{O} 1^{\mathrm{iv}}$ & $102.11(9)$ & $\mathrm{H} 10 \mathrm{~A}-\mathrm{O} 10-\mathrm{H} 10 \mathrm{~B}$ & 107.7 \\
\hline $\mathrm{O} 3-\mathrm{Na} 2-\mathrm{O}^{\mathrm{v}}$ & 180.0 & H11A-O11-H11B & 109.5 \\
\hline $\mathrm{O} 3^{\mathrm{v}}-\mathrm{Na} 2-\mathrm{O}^{\mathrm{iv}}$ & $98.25(9)$ & $\mathrm{H} 12 \mathrm{~A}-\mathrm{O} 12-\mathrm{H} 12 \mathrm{~B}$ & 107.7 \\
\hline $\mathrm{C} 1-\mathrm{C} 2-\mathrm{C} 3-\mathrm{C} 4$ & $0.2(5)$ & $\mathrm{C} 4-\mathrm{C} 5-\mathrm{C} 6-\mathrm{C} 1$ & $2.3(5)$ \\
\hline $\mathrm{C} 1-\mathrm{C} 2-\mathrm{C} 3-\mathrm{C} 8$ & $179.1(3)$ & $\mathrm{C} 4-\mathrm{C} 5-\mathrm{C} 9-\mathrm{O} 5$ & $-0.8(5)$ \\
\hline $\mathrm{C} 1-\mathrm{C} 7-\mathrm{O} 1-\mathrm{Zn} 1$ & $178.2(2)$ & $\mathrm{C} 4-\mathrm{C} 5-\mathrm{C} 9-\mathrm{O} 6$ & $-179.8(3)$ \\
\hline $\mathrm{C} 1-\mathrm{C} 7-\mathrm{O} 1-\mathrm{Na} 2^{\mathrm{i}}$ & $-48.0(5)$ & $\mathrm{C} 6-\mathrm{C} 1-\mathrm{C} 2-\mathrm{C} 3$ & $1.5(5)$ \\
\hline
\end{tabular}




$\begin{array}{llll}\mathrm{C} 1-\mathrm{C} 7-\mathrm{O} 2-\mathrm{Na} 1 & 114.6(3) & \mathrm{C} 6-\mathrm{C} 1-\mathrm{C} 7-\mathrm{O} 1 & -8.3(5) \\ \mathrm{C} 2-\mathrm{C} 1-\mathrm{C} 6-\mathrm{C} 5 & -2.8(5) & \mathrm{C} 6-\mathrm{C} 1-\mathrm{C} 7-\mathrm{O} 2 & 171.6(3) \\ \mathrm{C} 2-\mathrm{C} 1-\mathrm{C} 7-\mathrm{O} 1 & 170.3(3) & \mathrm{C} 6-\mathrm{C} 5-\mathrm{C} 9-\mathrm{O} 5 & 178.5(3) \\ \mathrm{C} 2-\mathrm{C} 1-\mathrm{C} 7-\mathrm{O} 2 & -9.9(5) & \mathrm{C} 6-\mathrm{C} 5-\mathrm{C} 9-\mathrm{O} 6 & -0.4(5) \\ \mathrm{C} 2-\mathrm{C} 3-\mathrm{C} 4-\mathrm{C} 5 & -0.7(5) & \mathrm{C} 7-\mathrm{C} 1-\mathrm{C} 2-\mathrm{C} 3 & -177.0(3) \\ \mathrm{C} 2-\mathrm{C} 3-\mathrm{C} 8-\mathrm{O} 3 & -178.0(3) & \mathrm{C} 7-\mathrm{C} 1-\mathrm{C} 6-\mathrm{C} 5 & 175.7(3) \\ \mathrm{C} 2-\mathrm{C} 3-\mathrm{C} 8-\mathrm{O} 4 & 0.4(5) & \mathrm{C} 8-\mathrm{C} 3-\mathrm{C} 4-\mathrm{C} 5 & -179.6(3) \\ \mathrm{C} 3-\mathrm{C} 4-\mathrm{C} 5-\mathrm{C} 6 & -0.6(5) & \mathrm{C} 9-\mathrm{C} 5-\mathrm{C} 6-\mathrm{C} 1 & -177.1(3) \\ \mathrm{C} 3-\mathrm{C} 4-\mathrm{C} 5-\mathrm{C} 9 & 178.8(3) & \mathrm{O} 1-\mathrm{C} 7-\mathrm{O} 2-\mathrm{Na} 1 & -65.6(4) \\ \mathrm{C} 3-\mathrm{C} 8-\mathrm{O} 3-\mathrm{Na} 2 & 116.2(3) & \mathrm{O} 2-\mathrm{C} 7-\mathrm{O} 1-\mathrm{Zn} 1 & -1.6(5) \\ \mathrm{C} 3-\mathrm{C} 8-\mathrm{O} 4-\mathrm{Zn} 1^{\mathrm{iv}} & -175.2(2) & \mathrm{O} 2-\mathrm{C} 7-\mathrm{O} 1-\mathrm{Na} 2^{\mathrm{i}} & 132.1(3) \\ \mathrm{C} 4-\mathrm{C} 3-\mathrm{C} 8-\mathrm{O} 3 & 0.9(5) & \mathrm{O} 3-\mathrm{C} 8-\mathrm{O} 4-\mathrm{Zn} 1^{\mathrm{iv}} & 3.1(4) \\ \mathrm{C} 4-\mathrm{C} 3-\mathrm{C} 8-\mathrm{O} 4 & 179.3(3) & \mathrm{O} 4-\mathrm{C} 8-\mathrm{O} 3-\mathrm{Na} 2 & -62.1(5)\end{array}$

Symmetry codes: (i) $x, y+1, z$; (ii) $-x+1,-y+2,-z$; (iii) $-x+1,-y+1,-z+1$; (iv) $x, y-1, z$; (v) $-x+1,-y,-z+1$.

Hydrogen-bond geometry $\left(A,{ }^{\circ}\right)$

\begin{tabular}{|c|c|c|c|c|}
\hline$D-\mathrm{H} \cdots A$ & $D-\mathrm{H}$ & $\mathrm{H} \cdots A$ & $D^{\cdots} A$ & $D-\mathrm{H} \cdots A$ \\
\hline $\mathrm{O} 7-\mathrm{H} 7 A \cdots \mathrm{O} 5^{\mathrm{vi}}$ & 0.82 & 1.79 & $2.587(4)$ & 162 \\
\hline $\mathrm{O} 7-\mathrm{H} 7 B^{\cdots} \cdots \mathrm{O} 12^{\mathrm{vii}}$ & 0.82 & 1.93 & $2.740(4)$ & 172 \\
\hline $\mathrm{O} 8-\mathrm{H} 8 A \cdots \mathrm{O} 10$ & 0.82 & 2.40 & $3.114(5)$ & 146 \\
\hline $\mathrm{O} 8-\mathrm{H} 8 A^{\cdots} \mathrm{O} 11$ & 0.82 & 1.98 & $2.672(8)$ & 142 \\
\hline $\mathrm{O} 8-\mathrm{H} 8 B \cdots \mathrm{O} 6^{\mathrm{vii}}$ & 0.82 & 2.05 & $2.641(5)$ & 128 \\
\hline $\mathrm{O} 9-\mathrm{H} 9 A^{\cdots} \cdots \mathrm{O} 12^{\text {viii }}$ & 0.82 & 1.95 & $2.734(4)$ & 159 \\
\hline $\mathrm{O} 9-\mathrm{H} 9 B^{\cdots} \mathrm{O} 2^{\mathrm{ix}}$ & 0.82 & 2.01 & $2.823(4)$ & 170 \\
\hline $\mathrm{O} 10-\mathrm{H} 10 A \cdots \mathrm{O} 5^{\mathrm{iii}}$ & 0.82 & 2.06 & $2.719(6)$ & 137 \\
\hline $\mathrm{O} 10-\mathrm{H} 10 B \cdots \mathrm{O} 9^{x}$ & 0.82 & 2.31 & $3.079(5)$ & 155 \\
\hline $\mathrm{O} 11-\mathrm{H} 11 A \cdots \mathrm{O} 3^{\mathrm{xi}}$ & 0.85 & 2.03 & $2.835(8)$ & 157 \\
\hline $\mathrm{O} 11-\mathrm{H} 11 B \cdots \mathrm{O} 3^{\mathrm{iii}}$ & 0.85 & 2.27 & $2.866(7)$ & 127 \\
\hline $\mathrm{O} 11-\mathrm{H} 11 B \cdots \mathrm{O} 11^{\mathrm{xii}}$ & 0.85 & 1.33 & $1.973(9)$ & 128 \\
\hline $\mathrm{O} 12-\mathrm{H} 12 A \cdots \mathrm{O} 6$ & 0.82 & 1.86 & $2.652(4)$ & 161 \\
\hline $\mathrm{O} 12-\mathrm{H} 12 B \cdots \mathrm{O} 4^{\mathrm{xiii}}$ & 0.82 & 1.97 & $2.787(3)$ & 172 \\
\hline
\end{tabular}

Symmetry codes: (iii) $-x+1,-y+1,-z+1$; (vi) $x, y+1, z-1$; (vii) $-x+1,-y+2,-z+1$; (viii) $-x+2,-y+2,-z+1$; (ix) $-x+2,-y+2,-z$; (x) $x-1, y, z$; (xi) $x-1$, $y+1, z$; (xii) $-x,-y+2,-z+1$; (xiii) $x, y, z+1$. 